\title{
Revista Colombiana de

\section{Reporte del primer caso de implante exitoso de asistencia ventricular izquierda HeartMate II como terapia de destino en Suramérica}

\author{
Antonio Figueredo ${ }^{\mathrm{a}, \mathrm{b}, *}$, Leonardo Salazar ${ }^{\mathrm{a}, \mathrm{b}}$, Luis E. Echeverría ${ }^{\mathrm{a}, \mathrm{c}}$, \\ Camilo Pizarro ${ }^{\mathrm{a}, \mathrm{d}}$ y Adriana S. Murcia ${ }^{\mathrm{a}, \mathrm{e}}$
}

a Departamento de Cirugía Cardiovascular, Fundación Cardiovascular de Colombia, Floridablanca, Santander, Colombia

b Cirugía Cardiovascular, Fundación Cardiovascular de Colombia, Floridablanca, Santander, Colombia

c Programa de Asistencia Ventricular. Fundación Cardiovascular de Colombia, Floridablanca, Santander, Colombia

d Departamento de Cuidados Intensivos. Fundación Cardiovascular de Colombia, Floridablanca, Santander, Colombia

e Departamento de Investigaciones. Fundación Cardiovascular de Colombia, Floridablanca, Santander, Colombia

Recibido el 2 de septiembre de 2015; aceptado el 21 de abril de 2016

Disponible en Internet el 6 de junio de 2016

\section{PALABRAS CLAVE \\ Dispositivo de asistencia cardiaca; Insuficiencia cardiaca; \\ Cirugía cardiaca}

\section{KEYWORDS}

Cardiac assist device; Heart failure;

Cardiac surgery
Resumen Se describe el caso de una paciente de sexo femenino de 55 años de edad, con insuficiencia cardiaca congestiva descompensada secundaria a cardiopatía dilatada idiopática y con contraindicación para trasplante cardiaco, en quien se implanta de manera exitosa el primer dispositivo de asistencia ventricular izquierda HeartMate ॥ en Suramérica.

(c) 2016 Sociedad Colombiana de Cardiología y Cirugía Cardiovascular. Publicado por Elsevier España, S.L.U. Este es un artículo Open Access bajo la licencia CC BY-NC-ND (http:// creativecommons.org/licenses/by-nc-nd/4.0/).

Report of the first successful case of HeartMate ॥ Left Ventricular Assist Device placement as a destination therapy in South America

Abstract The case of a 55 year-old woman with decompensated congestive heart failure secondary to idiopathic dilated cardiomyopathy is described. Cardiac transplantation was contraindicated, and the first HeartMate ॥ Left Ventricular Assist Device was placed in South America.

(c) 2016 Sociedad Colombiana de Cardiología y Cirugía Cardiovascular. Published by Elsevier España, S.L.U. This is an open access article under the CC BY-NC-ND license (http:// creativecommons.org/licenses/by-nc-nd/4.0/).

* Autor para correspondencia.

Correo electrónico: antoniofigueredo@fcv.org (A. Figueredo). 


\section{Introducción}

Hasta hace una década, la única terapia definitiva aprobada para los pacientes con insuficiencia cardiaca avanzada refractaria al manejo era el trasplante cardiaco ${ }^{1}$. No obstante, debido a: la baja cantidad de donantes, al mayor número de potenciales receptores por aumento en el diagnóstico y la coexistencia de algunas patologías que hacen a los candidatos no elegibles para un trasplante, relacionadas con la inmunosupresión y la necesidad de recuperación antes del procedimiento, se han desarrollado terapias alternativas al trasplante cardiaco ${ }^{2}$. Es así, como la implementación de los dispositivos de asistencia ventricular izquierda (Left Ventricular Assist Device, LVAD) en diferentes contextos clínicos, han permitido su aprobación como terapia de destino, es decir, una solución mecánica permanente para pacientes con insuficiencia cardiaca terminal que no son elegibles para trasplante cardiaco o donde este procedimiento no está disponible $e^{3,4}$.

Se presenta el primer caso de implante de dispositivo de asistencia ventricular HeartMate ॥ como terapia de destino en Suramérica y seguimiento a un año, en una paciente con insuficiencia cardiaca severa y contraindicación para trasplante cardiaco.

\section{Reporte de caso}

Paciente femenina de 55 años de edad, procedente de Barbosa, Santander, Colombia, ocupación: docente pensionada por enfermedad, con insuficiencia cardiaca congestiva desde el año 2008 secundaria a cardiopatía dilatada idiopática. Antecedentes de: hipotiroidismo, implante de cardiodesfibrilador en el año 2010 para prevención de muerte súbita, accidente cerebrovascular isquémico en enero de 2013 con secuelas de disartria leve y paresia mínima de miembro superior izquierdo, anticoagulación crónica por warfarina. En valoraciones por la institución desde julio de 2013 por insuficiencia cardiaca congestiva descompensada en clase funcional iv en estudio para trasplante cardiaco. Peso $40 \mathrm{~kg}$. Talla 1,55 m. Hemodinamia reporta: cardiopatía dilatada, FEVI 35\%, FEVD 33\%, insuficiencia mitral grado $\|$ y tricuspídea grado III, coronarias sanas, cateterismo derecho con hipertensión pulmonar moderada con presión sistólica arterial pulmonar $61 \mathrm{mmHg}$, gradiente transpulmonar $6 \mathrm{mmHg}$, y resistencias levemente elevadas.

El ecocardiograma transtorácico evidencia: cardiopatía dilatada, hipoquinesia difusa severa y disfunción sistólica y diastólica severas del ventrículo izquierdo, FEVI 15-18\%, dilatación auricular izquierda sin trombos, insuficiencia mitral grado Iv, insuficiencia pulmonar trivial, insuficiencia tricuspídea severa con hipertensión pulmonar moderada con PSAP $60 \mathrm{mmHg}$, cavidades derechas dilatadas con alteración en la motilidad de la pared libre, electrodos de desfibrilador en cavidades derechas, desplazamiento sistólico del plano del anillo tricuspídeo (siglas en inglés, TAPSE) $11 \mathrm{~mm}$. Péptido atrial natriurético (siglas en inglés, BNP) de $844 \mathrm{pg} / \mathrm{ml}$.

Consumo pico de oxígeno $10,5 \mathrm{ml} / \mathrm{kg} / \mathrm{min}$. Test de Chagas negativo. PRA (Panel Reactivity Antibody) cuantitativo Clase I 56\%, PRA Clase 2 del 63\%. Serología positiva para HTLV (Human T-lymphotropic virus) tipo I y $॥$, que contraindica el trasplante cardiaco debido a la posibilidad de desarrollar leucemia de células T. Se considera candidata para recibir un LVAD como terapia de destino (HeartMate ॥).

En la tabla 1 se muestra la estratificación de la insuficiencia cardiaca congestiva de la paciente según la escala INTERMACS (Interagency Registry for Mechanically Assisted Circulatory Support $)^{5}$, que arroja puntaje 4 y permite el tiempo a la intervención en semanas o meses. Pero dado que la paciente a pesar de estar recibiendo manejo óptimo para

Tabla 1 Escala INTERMACS (Interagency Registry for Mechanically Assisted Circulatory Support) para la clasificación de los pacientes con insuficiencia cardiaca avanzada

\begin{tabular}{|c|c|c|}
\hline Clasificación & Descripción & Tiempo a la Intervención \\
\hline INTERMACS 1 & $\begin{array}{l}\text { Inestabilidad hemodinámica pese a dosis crecientes de } \\
\text { catecolaminas y/o soporte circulatorio mecánico con } \\
\text { hipoperfusión crítica de órganos diana (shock cardiogénico } \\
\text { crítico). }\end{array}$ & En horas. \\
\hline INTERMACS 2 & $\begin{array}{l}\text { Soporte inotrópico intravenoso con cifras aceptables de } \\
\text { presión arterial y deterioro rápido de la función renal, el } \\
\text { estado nutricional o los signos de congestión. }\end{array}$ & En días. \\
\hline INTERMACS 3 & $\begin{array}{l}\text { Estabilidad hemodinámica con dosis bajas o intermedias de } \\
\text { inotrópicos e imposibilidad para su retirada por hipotensión, } \\
\text { empeoramiento sintomático o insuficiencia renal progresiva. }\end{array}$ & Electivo en semanas o meses. \\
\hline INTERMACS 4 & $\begin{array}{l}\text { Es posible retirar transitoriamente el tratamiento inotrópico, } \\
\text { pero el paciente presenta recaídas sintomáticas frecuentes, } \\
\text { habitualmente con sobrecarga hídrica. }\end{array}$ & Electivo en semanas o meses. \\
\hline INTERMACS 5 & $\begin{array}{l}\text { Limitación absoluta de la actividad física, con estabilidad en } \\
\text { reposo, aunque habitualmente con retención hídrica } \\
\text { moderada y un cierto grado de disfunción renal. }\end{array}$ & $\begin{array}{l}\text { Urgencia variable, dependiendo de la } \\
\text { nutrición y función de los órganos. }\end{array}$ \\
\hline INTERMACS 6 & $\begin{array}{l}\text { Menor limitación de la actividad física y ausencia de } \\
\text { congestión en reposo. Fatiga fácil con actividad ligera. }\end{array}$ & $\begin{array}{l}\text { Urgencia variable, dependiendo de la } \\
\text { nutrición y función de los órganos. }\end{array}$ \\
\hline INTERMACS 7 & $\begin{array}{l}\text { Paciente en clase funcional NYHA ॥-II sin balance hídrico } \\
\text { inestable actual ni reciente. }\end{array}$ & Actualmente no indicado. \\
\hline
\end{tabular}


Tabla 2 Escala Lietz-Miller DTRS (Destination Therapy Risk Score) para evaluación preoperatoria de soporte circulatorio mecánico definitivo

\begin{tabular}{|c|c|c|c|}
\hline \multicolumn{3}{|l|}{ Variable } & Puntaje \\
\hline \multicolumn{3}{|l|}{ Plaquetas $<148,000 \mathrm{ml}$} & 7 \\
\hline \multicolumn{3}{|c|}{ Albúmina sérica $\leq 3,3 \mathrm{~g} / \mathrm{dl}$} & 5 \\
\hline \multicolumn{3}{|c|}{ INR $>1,1$} & 4 \\
\hline \multicolumn{3}{|c|}{ Vasodilatador } & 4 \\
\hline \multicolumn{3}{|c|}{ Presión arterial media pulmonar $\leq 25 \mathrm{mmHg}$} & 3 \\
\hline \multicolumn{3}{|c|}{ Aspartato aminotransferasa $>45 \mathrm{U} / \mathrm{ml}$} & 2 \\
\hline \multicolumn{3}{|c|}{ Hematocrito $\leq 34 \%$} & 2 \\
\hline \multicolumn{3}{|c|}{ Nitrógeno ureico > $51 \mathrm{U} / \mathrm{dl}$} & 2 \\
\hline \multicolumn{3}{|c|}{ Sin inotrópicos intravenosos } & 2 \\
\hline \multirow[t]{2}{*}{ Sumatoria del Puntaje } & \multicolumn{3}{|c|}{ Sobrevida, \% } \\
\hline & Egreso & 90 días & 1 año \\
\hline 0-8: Bajo & 87,5 & 93,7 & 81,2 \\
\hline 9-16: Medio & 70,5 & 86,5 & 62,4 \\
\hline 17-19: Alto & 26,0 & 38,9 & 27,8 \\
\hline >19: Muy Alto & 13,7 & 17,9 & 10,7 \\
\hline
\end{tabular}

falla cardiaca presentó más de dos hospitalizaciones en los últimos seis meses y su clase funcional se deterioraba progresivamente presentando disnea de mínimos esfuerzos, se decidió el implante de HeartMate I. En la tabla 2 se muestra la evaluación preoperatoria de soporte circulatorio mecánico definitivo según la escala Lietz-Miller DTRS (Destination Therapy Risk Score $)^{6}$, que clasifica la paciente con puntaje 6 , y predice porcentajes de sobrevida con el LVAD al egreso de $87 \%$, a los 90 días de $93 \%$, y al año de $81 \%$.

El 7 de abril de 2014, se realiza el implante de HeartMate II (Thoratec Corporation, Pleasanton, CA, USA), dispositivo de segunda generación, de flujo axial continuo, mediante esternotomía mediana, en corazón batiente y usando bypass cardiopulmonar transitorio y técnicas de ahorro sanguíneo. El dispositivo tiene una cánula de entrada de titanio que se implanta en el ápex del ventrículo izquierdo, que tiene continuidad con el cuerpo de la bomba que se aloja en un bolsillo creado entre el diafragma y la fascia del recto anterior, y que continúa con la porción de salida de la bomba, constituida por un injerto de dacron que se anastomosa a la aorta ascendente (fig. 1). Se monitorizó con un catéter continuo de gasto cardiaco y saturación. Se extubó a las 24 horas, pero se reintubó por efecto medicamentoso anestésico residual. Se extuba definitivamente a los dos días. Seis días después del implante es llevada a reoperación por derrame pericárdico de $400 \mathrm{cc}$ secundario a sobreanticoagulación sin evidencia de sangrado activo y con cánulas en adecuada posición.

Permanece hospitalizada por 40 días, 16 en cuidados intensivos y 24 en hospitalización general, por dificultad en el proceso de anticoagulación, con labilidad marcada y episodios de sobreanticoagulación sintomática dada por epistaxis, y otros de subanticoagulación. El uso del monitoreo guiado con tromboelastografía y mapeo plaquetario fue útil en determinar cuando debía comenzarse la terapia anticoagulante. El cardiodesfibrilador se reprograma y se ordena seguimiento ambulatorio cada tres meses.

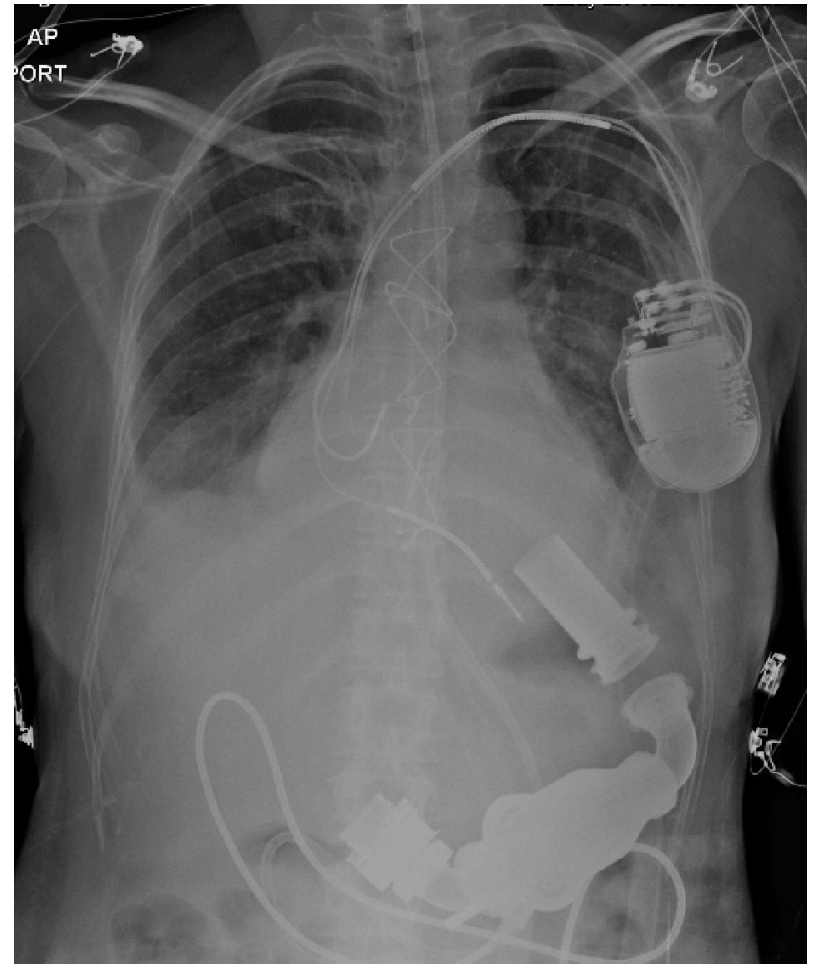

Figura 1 Radiografía de la paciente que muestra los componentes de HeartMate $\|$ y el cardiodesfibrilador previamente implantado.

El plan de anticoagulación se estableció según las indicaciones del fabricante del dispositivo y las recomendaciones publicadas en la literatura médica ${ }^{7-9}$. Durante las primeras 24 horas postoperatorias no se utiliza anticoagulación. Se inicia con heparina no fraccionada cuando el drenaje por tubos es menor de $50 \mathrm{cc}$ en cuatro horas y la tromboelastografía es normal. Se busca una meta de PTT (tiempo parcial de tromboplastina) entre 45-60 segundos el primer día de inicio de anticoagulación y entre $60-90$ segundos el segundo día. Al cuarto día de anticoagulación se inicia con aspirina $100 \mathrm{mg} /$ día y tres días después se controla con mapeo plaquetario para obtener una antiagregación entre el 70 y $95 \%$. Esta paciente tenía resistencia a la acción de la aspirina y se antiagregó finalmente con el clopidogrel, confirmándose por mapeo plaquetario una antiagregación adecuada. Después de retirarse los tubos de mediastino se inicia con la warfarina para obtener un INR entre 2-3. Cuando el INR es mayor de 2 se suspende definitivamente la heparina. Al egreso la paciente se manejó con la warfarina $2,5 \mathrm{mg} /$ día más el clopidogrel $75 \mathrm{mg} /$ día. Este plan de anticoagulación es permanente.

Egresa en buenas condiciones generales, en clase funcional NYHA II, y tolerando el plan escalonado de rehabilitación cardiovascular de 30 sesiones. La paciente y su familia fueron educados en la rutina diaria del manejo de los componentes externos del dispositivo como son: la miniconsola, los conductores eléctricos y las baterías; así como en la respuesta oportuna a las potenciales situaciones de emergencias, como: fallos en el suministro eléctrico, desconexiones, respuesta a las alarmas y cuidado diario del 
conductor percutáneo, que inicialmente fue supervisado por el equipo médico hasta aprobar total autonomía.

Antes de la implantación del dispositivo, el paciente y su familia recibieron cinco sesiones de entrenamiento durante una semana a cargo del director del programa de asistencia ventricular y el enfermero coordinador, donde se revisaron: la indicación por el estado de la falla cardiaca, el funcionamiento básico y habitual del aparato, y el manejo de complicaciones. Al finalizar las cinco sesiones la paciente y su familia firmaron el consentimiento informado y se programó el procedimiento. Durante la hospitalización la paciente recibió 20 horas de entrenamiento referentes: al cambio de baterías, al cuidado del equipo, a la realización de las curaciones del conductor eléctrico y al manejo de emergencias. Los últimos días de hospitalización la paciente realizó salidas de la institución bajo supervisión médica para confirmar el nivel de confianza y confort con todo el sistema. Antes del egreso la paciente y su familia debieron realizar una maniobra de cambio de controlador por ellos mismos. También se realizó una visita domiciliaria con la paciente para confirmar la seguridad eléctrica y las condiciones generales de seguridad de la casa.

Durante el entrenamiento se reforzaron las contraindicaciones absolutas para sumergirse (el dispositivo no es impermeable) y para realizarse resonancias magnéticas nucleares. La paciente recibió entrenamiento en el aseo en ducha con bolsa impermeable para el dispositivo. La paciente puede ingerir bebidas alcohólicas con moderación, pero tiene contraindicada la embriaguez por la disminución en la habilidad motriz para manejar el cambio de baterías y las posibles emergencias.

Para garantizar la seguridad en el suministro eléctrico, se evaluó la estabilidad de las conexiones y el polo a tierra en la residencia de la paciente y los sitios alternativos de recarga en su municipio de origen, así como la disponibilidad permanente del acceso a la planta eléctrica en el hospital regional local. También se entrenó al equipo de urgencias de este hospital en el conocimiento del dispositivo y el manejo médico inicial de las posibles complicaciones.

A partir del egreso se realizaron controles mensuales de: revisión de los signos de falla cardiaca, verificación de los índices de pulsatilidad, amperaje, revoluciones, ocurrencia de eventos de succión, pruebas de coagulación y deshidrogenasa láctica, que se encontraron en los parámetros esperados. Los primeros seis meses se realizan controles mensuales y después cada dos meses. A partir del segundo año los controles se realizan cada seis meses y después del cuarto año, de forma anual. El control ambulatorio a largo plazo consiste en interrogar el dispositivo con una consola de control que permanece en el hospital para revisar si se han presentado eventos, control del sitio de salida del conductor eléctrico para descartar infecciones, toma de presión arterial con doppler y evaluación de signos y síntomas de falla cardiaca o sangrado secundario a la anticoagulación. A los 22 meses de seguimiento ambulatorio, la paciente permanece asintomática en clase funcional NYHA I, autosuficiente, manteniendo los niveles de INR entre 2-3 con la warfarina, control de BNP de $47 \mathrm{pg} / \mathrm{ml}$, velocidad de la bomba establecida a $9.200 \mathrm{rpm}$ donde el ventrículo se ve de diámetro $3,5 \mathrm{~cm}$, sin desplazamiento del septo y sin apertura de la válvula aórtica, flujo de 6 litros, poder de $7 \mathrm{~W}$, e índice de pulsatilidad 4,9. La prueba EuroQol-5D, un instrumento genérico para medir la calidad de vida en salud y que se ha utilizado en receptores de soporte circulatorio mecánico ${ }^{10}$, arrojó un resultado óptimo en cuatro de sus cinco áreas: cuidado personal, sensación de dolor o malestar, sensación de angustia o depresión y movilidad, mientras para las actividades cotidianas mostró un resultado promedio.

\section{Discusión}

El soporte circulatorio mecánico se derivó del desarrollo de la circulación extracorpórea e incluye diferentes dispositivos para proveer asistencia de corto, mediano y largo plazo en pacientes con falla cardiaca crónica y aguda ${ }^{11}$. Esta tecnología se puede utilizar como: puente a decisión, puente a trasplante, puente a recuperación, terapia de destino y puente a otra terapia de mayor duración ${ }^{12-14}$. La tendencia actual son: los dispositivos más pequeños, seguros y durables y totalmente implantables con recarga de energía transcutánea ${ }^{15}$.

En los pacientes que cumplen los siguientes criterios está indicada la implantación de estos dispositivos: pacientes con falla cardiaca que han recibido por lo menos seis meses de tratamiento óptimo de la falla cardiaca, y que en esos seis meses han requerido dos o más hospitalizaciones por descompensación de la misma o que presentan disminución de la clase funcional que les impide caminar más de una cuadra a pesar del máximo manejo médico, o que no sea posible titularles por intolerancia los inhibidores de la enzima convertidora de angiotensina y los betabloqueadores. Estos pacientes tienen un riesgo estimado muy alto de mortalidad a un año y deben evaluarse inicialmente para trasplante cardiaco o para asistencia ventricular de largo término como segunda opción ${ }^{16}$.

HeartMate XVE fue el primer dispositivo aprobado por la FDA (Food and Drug Administration) en el año 2003 como terapia de destino, basados en el estudio REMATCH (Randomized Evaluation of Mechanical Assistance for the Treatment of Congestive Heart Failure Trial) que demostró una reducción del $48 \%$ de mortalidad con LVAD comparado con el manejo médico óptimo en pacientes no elegibles para trasplante cardiaco ${ }^{17}$.

El HeartMate ॥ LVAS es un sistema de asistencia ventricular izquierda, rotatorio y de flujo axial capaz de generar flujos de hasta 10 litros por minuto (lpm). La bomba de sangre HeartMate "I se conecta al ápice del ventrículo izquierdo y la aorta ascendente, desvía la sangre del ventrículo izquierdo debilitado y la impulsa hacia el resto del cuerpo. La miniconsola del sistema, mediante su programa informático interno, regula la bomba (fig. 2).

El primer reporte de implante de HeartMate " como puente a trasplante se publicó en el año $2004^{18}$, logrando la aprobación de la FDA para esta indicación en el año 2008. En el año 2010 la FDA aprueba el HeartMate ॥ como terapia de destino, basados en el HeartMate ॥ DT Trial, que demostró una sobrevida con el HeartMate ॥ de $68 \%$ al año y $58 \%$ a los 2 años, comparado con 52\% al año y $24 \%$ a los dos años con el HeartMate XVE. Además, la sobrevida libre de enfermedad cerebrovascular, la disfunción del dispositivo y la necesidad de reoperación a los dos años de seguimiento fue del $48 \%$ para el HeartMate ॥ comparado con $7 \%$ para los LVAD de flujo pulsátil ${ }^{19}$. 


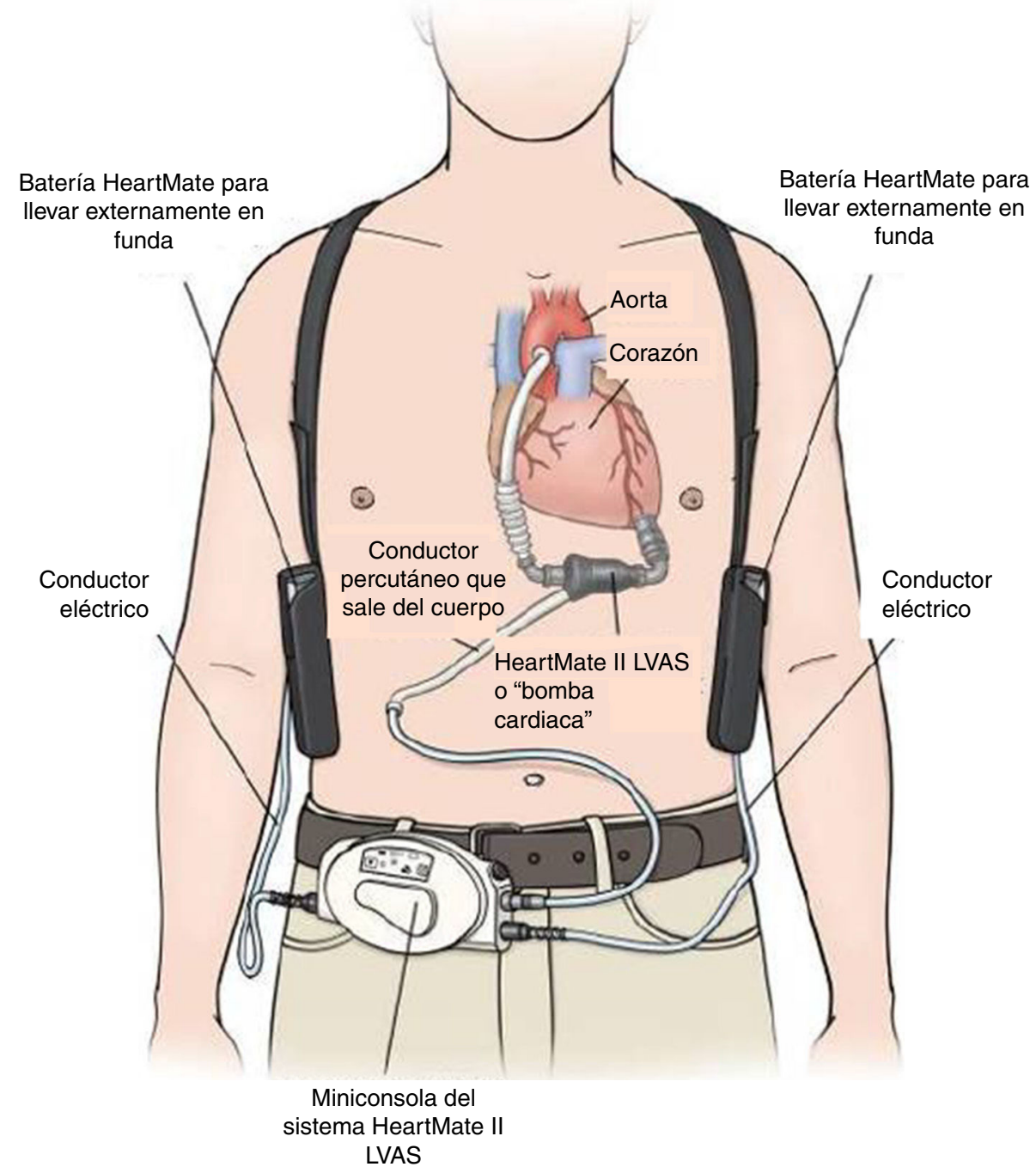

Figura 2 Componentes del HeartMate II. (Tomado de HeartMate ॥ Sistema de Asistencia Ventricular Izquierda. Manual de Operación. Thoratec Corporation).

El beneficio del tratamiento con el HeartMate ॥ como terapia de destino se ha confirmado en estudios posteriores, con mejores tasas de eventos adversos y una fuerte tendencia en el mejoramiento de la supervivencia ${ }^{20}$.

En cuanto a las complicaciones en la implantación de estos dispositivos ${ }^{21,22}$, se describe el sangrado que requiere cirugía en $20 \%$ de los pacientes ${ }^{20}$. La paciente que nos ocupa, presentó un cuadro de epistaxis y taponamiento cardiaco secundario a sobreanticoagulación. Resaltamos la utilidad de la tromboelastografía en la monitorización de los eventos de sobreanticoagulación y subanticoagulación, que son un asunto crítico en el postoperatorio de estos pacientes.

Es importante recalcar que el manejo seguro del dispositivo es elemental para garantizar el éxito de la terapia a largo plazo. Por tanto, debe destinarse el tiempo necesario en los períodos preoperatorio y postoperatorio para que el paciente y su familia, interioricen con suficiencia los conocimientos y habilidades necesarias para el manejo cotidiano del dispositivo ${ }^{23,24}$.
Para nuestro conocimiento, y luego de una búsqueda bibliográfica exhaustiva en las bases de datos PubMed, SciELO, Lilacs y Bireme, y de consultar directamente a Thoratec Corporation, Pleasanton, CA, USA, fabricante del dispositivo, el caso que presentamos es el primer implante de HeartMate ॥ en Suramérica. El segundo paciente que recibió este dispositivo en Suramérica fue un niño en Chile en octubre de 2014, seis meses después del implante realizado por nuestro grupo quirúrgico, del que no se encontró reporte en la bibliografía médica. (Primer implante de corazón artificial pediátrico en Chile y Sudamérica. http: / / www. reich.cl/2014/10/30/primer-implante-de-corazon-artificialpediatrico-en-chile-y-sudamerica/. Accesado 02 de marzo de 2016). El caso publicado en el año 2012 en Argentina corresponde a un paciente operado en Estados Unidos ${ }^{25}$. El artículo publicado en el año 2014 en Chile corresponde a un paciente que recibió el implante del HeartMate ॥ en el año 2010 en Estados Unidos y que en el año 2011 se trasladó a Chile donde realizan el trasplante cardiaco $^{26}$. 


\section{Conclusión}

Presentamos el primer caso en Suramérica de un implante de dispositivo de asistencia ventricular izquierdo no pulsátil, como terapia de destino en una paciente con insuficiencia cardiaca no elegible para trasplante cardiaco. En más de un año de seguimiento la paciente se mantiene en clase funcional NYHA I y óptima calidad de vida. Consideramos que esta tecnología como terapia de destino es una opción que crecerá en nuestra región ajustándose a las recomendaciones y criterios que han sido establecidos en latitudes con amplia experiencia.

\section{Responsabilidades éticas}

Protección de personas y animales. Los autores declaran que los procedimientos seguidos se conformaron a las normas éticas del comité de experimentación humana responsable y de acuerdo con la Asociación Médica Mundial y la Declaración de Helsinki.

Confidencialidad de los datos. Los autores declaran que han seguido los protocolos de su centro de trabajo sobre la publicación de datos de pacientes.

Derecho a la privacidad y consentimiento informado. Los autores han obtenido el consentimiento informado de los pacientes y/o sujetos referidos en el artículo. Este documento obra en poder del autor de correspondencia.

\section{Fuentes de Apoyo}

Institucional

\section{Conflicto de intereses}

Los autores declaran no tener ningún conflicto de intereses.

\section{Agradecimientos}

Al doctor Álvaro Javier Quintero. Médico interno. Universidad Autónoma de Bucaramanga.

\section{Bibliografía}

1. McMurray JJ, Adamopoulos S, Anker SD, Auricchio A, Böhm M, Dickstein $\mathrm{K}$, et al., ESC Guidelines for the diagnosis and treatment of acute and chronic heart failure 2012: The Task Force for the Diagnosis and Treatment of Acute and Chronic Heart Failure 2012 of the European Society of Cardiology. Developed in collaboration with the Heart Failure Association (HFA) of the ESC. Eur Heart J. 2012;33(14):1787-847.

2. Pérez de la Sota E. Asistencia circulatoria permanente en la insuficiencia cardiaca crónica refractaria. Cir. Cardiov. 2011;18(3):175-88.

3. Peura JL, Colvin-Adams M, Francis GS, Grady KL, Hoffman TM, Jessup $M$, et al. Recommendations for the use of mechanical circulatory support: device strategies and patient selection: a scientific statement from the American Heart Association. Circulation. 2012;126(22):2648-67.
4. Fang JC. Rise of the machines - left ventricular assist devices as permanent therapy for advanced heart failure. N Engl J Med. 2009;361(23):2282-5.

5. Stevenson LW, Pagani FD, Young JB, Jessup M, Miller L, Kormos $\mathrm{RL}$, et al. INTERMACS profiles of advanced heart failure: the current picture. J Heart Lung Transplant. 2009;28:535-41.

6. Lietz K, Long JW, Kfoury AG, Slaughter MS, Silver MA, Milano CA, et al. Outcomes of left ventricular assist device implantation as destination therapy in the post-REMATCH era: implications for patient selection. Circulation. 2007;116:497-505.

7. Amir O, Bracey AW, Smart FW, Delgado RM 3rd, Shah N, Kar B. A successful anticoagulation protocol for the first HeartMate II implantation in the United States. Tex Heart Inst J. 2005;32(3):399-401.

8. Baumann Kreuziger L, Massicotte MP. Mechanical circulatory support: balancing bleeding and clotting in high-risk patients. Hematology Am Soc Hematol Educ Program. 2015 Dec 5;2015(1):61-8.

9. Baumann Kreuziger LM. Management of anticoagulation and antiplatelet therapy in patients with left ventricular assist devices. J Thromb Thrombolysis. 2015 Apr;39(3):337-44.

10. Grady KL, Naftel DC, Myers S, Dew MA, Weidner G, Spertus $\mathrm{JA}$, et al. Change in health-related quality of life from before to after destination therapy mechanical circulatory support is similar for older and younger patients: analyses from INTERMACS. J Heart Lung Transplant. 2015 Feb;34(2):213-21.

11. Stewart GC, Givertz MM. Mechanical circulatory support for advanced heart failure: patients and technology in evolution. Circulation. 2012;125(10):1304-15.

12. Lahpor J, Khaghani A, Hetzer R, Pavie A, Friedrich I, Sander K, et al. European results with a continuous-flow ventricular assist device for advanced heart-failure patients. Eur J Cardiothorac Surg. 2010;37(2):357-61.

13. Pagani FD, Miller LW, Russell SD, Aaronson KD, John R, Boyle $\mathrm{AJ}$, et al. Extended mechanical circulatory support with a continuous-flow rotary left ventricular assist device. J Am Coll Cardiol. 2009;54(4):312-21.

14. Starling RC, Naka Y, Boyle AJ, Gonzalez-Stawinski G, John $\mathrm{R}$, Jorde $\mathrm{U}$, et al. Results of the post-U.S. Food and Drug Administration-approval study with a continuous flow left ventricular assist device as a bridge to heart transplantation: a prospective study using the INTERMACS (Interagency Registry for Mechanically Assisted Circulatory Support). J Am Coll Cardiol. 2011;57(19):1890-8.

15. Patel CB, Cowger JA, Zuckermann A. A contemporary review of mechanical circulatory support. J Heart Lung Transplant. 2014;33(7):667-74.

16. Feldman D, Pamboukian SV, Teuteberg JJ, Birks E, Lietz K, Moore SA, et al. The 2013 International Society for Heart and Lung Transplantation Guidelines for mechanical circulatory support: executive summary. J Heart Lung Transplant. 2013;32(2):157-87.

17. Rose EA, Gelijns AC, Moskowitz AJ, Heitjan DF, Stevenson LW, Dembitsky W, et al. Long-term use of a left ventricular assist device for end-stage heart failure. N Engl J Med. 2001;345:1435-43.

18. Frazier OH, Reynolds M, Delgado III, Kar B, Patel V, Gregoric I, et al. First Clinical Use of the Redesigned HeartMate ${ }^{\circ}$ II Left Ventricular Assist System in the United States: A Case Report. Tex Heart Inst J. 2004;31(2):157-9.

19. Slaughter MS, Rogers JG, Milano CA, Russell SD, Conte JV, Feldman D, et al. Advanced heart failure treated with continuous-flow left ventricular assist device. N Engl J Med. 2009;361:2241-51.

20. Park SJ, Milano CA, Tatooles AJ, Rogers JG, Adamson RM, Steidley DE, et al., HeartMate II Clinical Investigators. Outcomes in advanced heart failure patients with left ventricular assist devices for destination therapy. Circ Heart Fail. 2012;5(2):241-8. 
21. Genovese EA, Dew MA, Teuteberg JJ, Simon MA, Kay J, Siegenthaler MP, et al. Incidence and patterns of adverse event onset during the first 60 days after ventricular assist device implantation. Ann Thorac Surg. 2009;88(4): 1162-70.

22. Kirklin JK, Naftel DC, Pagani FD, Kormos RL, Stevenson LW, Blume ED, et al. Seventh INTERMACS annual report: 15,000 patients and counting. J Heart Lung Transplant. 2015;34(12):1495-504.

23. Kato N, Jaarsma T, Ben Gal T. Learning Self-care After Left Ventricular Assist Device Implantation. Curr Heart Fail Rep. 2014;11:290-8.
24. Ben Gal T, Jaarsma T. Self-care and communication issues at the end of life of recipients of a left-ventricular assist device as destination therapy. Curr Opin Support Palliat Care. 2013;7(1):29-35.

25. Levin R, Degrange M, Salvagio F, Blanco N, Botbol A, Porcile Rafael, et al. Asistencia ventricular sistémica con dispositivo HeartMate 2 como puente al trasplante en la transposición corregida de los grandes vasos. Rev Argent Cardiol. 2012;80:390-3

26. Bunster N, Villavicencio M, Lim J, Donoso E, Gajardo F, Rossel V. Primer puente a trasplante cardiaco exitoso en Chile usando el dispositivo Heart Mate II. Rev Med Chile. 2014. 2014;142:521-5. 